\title{
Response of fractionally damped beams with general boundary conditions subjected to moving loads
}

\author{
R. Abu-Mallouh ${ }^{\mathrm{a}, *}$, I. Abu-Alshaikh ${ }^{\mathrm{a}}$, H.S. Zibdeh ${ }^{\mathrm{b}}$ and Khaled Ramadan ${ }^{\mathrm{a}}$ \\ ${ }^{a}$ Applied Sciences University, Amman 11931, Jordan \\ ${ }^{\mathrm{b}}$ Jordan University of Science and Technology, Irbid, Jordan
}

Received 14 December 2009

Revised 1 November 2010

\begin{abstract}
This paper presents the transverse vibration of Bernoulli-Euler homogeneous isotropic damped beams with general boundary conditions. The beams are assumed to be subjected to a load moving at a uniform velocity. The damping characteristics of the beams are described in terms of fractional derivatives of arbitrary orders. In the analysis where initial conditions are assumed to be homogeneous, the Laplace transform cooperates with the decomposition method to obtain the analytical solution of the investigated problems. Subsequently, curves are plotted to show the dynamic response of different beams under different sets of parameters including different orders of fractional derivatives. The curves reveal that the dynamic response increases as the order of fractional derivative increases. Furthermore, as the order of the fractional derivative increases the peak of the dynamic deflection shifts to the right, this yields that the smaller the order of the fractional derivative, the more oscillations the beam suffers. The results obtained in this paper closely match the results of papers in the literature review.
\end{abstract}

Keywords: Fractional damping, moving load, vibration, beams, fractional derivative

\section{Introduction}

The general problem of predicting the transverse vibrations of continuous media resulting from the passage of moving loads is of considerable practical interest in the dynamics of structures. This problem has many applications in engineering systems, some of these applications are vibrations that occur in bridges and railroad tracks due to moving vehicles, vibrations that occur in pipe systems due to fluid flow, machining operations, machine chains and belt drives, and thermal processing subjected to moving heat sources.

This paper presents a homogeneous isotropic fractionally damped beam with general boundary conditions subjected to a moving load. The basic dynamic characteristics of the beam are obtained based on the assumption of the moving force model. This model is widely used when the mass of the moving system in comparison to the mass of the beam is sufficiently small. The solutions are obtained by decomposing the transverse displacement of the beam in the Laplace transform domain into an infinite series. The components of the series are then found recursively and the problem is solved by using the inverse Laplace transform of the decomposed equations. This method is able to get the infinite series solution directly by introducing the well known Mittag-Leffler function which is also known as "fractional exponential function". This solution can be written in a closed form for some special cases; otherwise, it may be truncated easily by using mathematical software like Maple. Similar ideas of decomposing dependent variables for solving nonlinear differential equations of various types are implemented in [1,2].

\footnotetext{
*Corresponding author. Tel.: +96 2788607797; E-mail: Riad_abumlwah@asu.edu.jo.
} 


\subsection{Literature review}

The vibration of solids and structures under moving loads involves many related problems. Many research papers were presented to solve and explain these problems [3-9]. Perhaps the most important problem is the one that deals with inherent randomness due to material properties like force, supports and speed. Other studies have been conducted on the extensions of rotating beams and Timoshenko beams [10-12]. Further studies have described the vibration response of beams on the elastic foundation due to a moving force [13,14]. The response of a simply supported beam subjected to a moving mass has been treated by using dynamic Green's function approach or by approximations based on model analysis $[15,16]$. The problem of an elastic system subjected to a moving oscillator has also been investigated in [17]. Other research work on the problem of transverse vibrations of homogeneous beams with general boundary conditions due to the passage of different types of loads, including the effects of internal and external damping, has been conducted in $[18,19]$. In all previews references, the damping characteristics whenever taken into consideration were assumed to be of first order derivative model. However, in this paper the problem is formulated using the fractional derivative model of an arbitrary order, proving that in the limiting case, the first derivative model is recovered.

Many physical problems were adequately described by differential equations of fractional order. Among these applications are polymeric damping, fluid mechanics, and theory of viscoelasticity [20-25]. However, as noted by these authors and from other literature, a clear physical interpretation of the fractional derivative has been elusive. Good examples are:

1) In the diffusion process, fractional differential equations have been employed to describe an anomalous diffusion regime, including both sub-diffusion and super-diffusion [26].

2) Many linear viscoelastic damping materials exhibit a macroscopic constitutive behavior, which has been the subject of many investigations involving fractional order derivative [27].

However, in the fractional model of viscoelastic material over extended ranges of time and frequency, the deformation work corresponds to springs and the losses to dashpots have both energy types(stored and dissipated) at any point of the material $[28,29]$. Transverse vibration of homogeneous beams whose damping behavior is described by a fractional derivative of arbitrary orders was an important subject of matter [30-32]. Further studies investigated the analytical scheme for stochastic analysis of a continuous beam whose damping characteristics are described by a fractional derivative model of order half [30]. The analytical solution of an initially stationary oscillator subjected to a unit step or impulse excitation were the damping is described by a fractional derivative model of order half was performed in [31]. In this analytical solution, Adomian decomposition method is applied. Other researchers investigated the analytical solution of a viscoelastic continuous beam whose damping characteristics are described in terms of fractional derivative of arbitrary order [32]. This problem was solved by the Adomian decomposition method and the beam is assumed to be subjected to a unit step or impulse function response.

\subsection{Formulation of the problem}

Transverse vibrations of Bernoulli-Euler homogeneous isotropic fractionally damped beams with general boundary conditions are investigated. The beams are assumed to be subjected to a load moving with a uniform velocity from left to right. Moreover, the beams are assumed to be of finite length and undisturbed before the load is applied (originally at rest). The governing equation of the beam under investigation whose damping characteristics are described by a fractional derivative of order $\beta$ can be written as

$$
E I \frac{\partial^{4} w(x, t)}{\partial x^{4}}+C \frac{\partial^{\beta} w(x, t)}{\partial t^{\beta}}+\mu \frac{\partial^{2} w(x, t)}{\partial t^{2}}=P(x, t),
$$

where $E, I, \mu$ and $C$ are, the modulus of elasticity, moment of inertia of cross-sectional area, mass per unit length, and the coefficient of external damping of the beam, respectively. It is assumed that $E, I, C$ and $\mu$ are constants. Furthermore, $P(x, t)$ is the applied moving load, and $w(x, t)$ is the transverse deflection of the beam at point $x$ and time $t$. 
The fractional derivative of order $\beta$ may be defined in many ways [33]. Among these are two most frequently encountered: the Riemann-Liouville and Caputo fractional derivatives of order $\beta$, which are defined, respectively, by the following convolution integrals [33].

$$
\begin{aligned}
& \frac{\partial^{\beta} w(x, t)}{\partial t^{\beta}}=\frac{1}{\Gamma(k-\beta)} \frac{\partial^{k}}{\partial t^{k}} \int_{0}^{t} \frac{w(x, u)}{(t-u)^{\beta+1-k}} d u \\
& \frac{\partial^{\beta} w(x, t)}{\partial t^{\beta}}=\frac{1}{\Gamma(k-\beta)} \int_{0}^{t} \frac{1}{(t-u)^{\beta+1-k}} \frac{\partial^{k} w(x, u)}{\partial u^{k}} d u
\end{aligned}
$$

where $k$ is a positive integer such that $k-1 \leqslant \beta<k$ and $\Gamma$ represents the Gamma function. The second term of Eq. (1), with the convolution integrals in Eqs (2-3), represent a proportional viscous damping model. However, there are other types of non - viscous damping that can be expressed in terms of convolution integrals like that used by Adhikari and Friswell, et al. [34,35].

In modal form, the transverse deflection of the beam is written as

$$
w(x, t)=\sum_{n=1}^{\infty} Y_{n}(t) X_{n}(x)
$$

where $Y_{n}(t)$ is the generalized displacement or the modal response of the beam, $X_{n}(x)$ are the normal modes of the undamped free vibration of the beam written as [18]

$$
X_{n}(x)=\sin \kappa_{n} x+A_{n} \cos \kappa_{n} x+B_{n} \sinh \kappa_{n} x+C_{n} \cosh \kappa_{n} x,
$$

which satisfy the following differential equation

$$
E I \frac{\partial^{4} X_{n}(x)}{\partial x^{4}} Y_{n}(t)+\mu \frac{\partial^{2} Y_{n}(t)}{\partial t^{2}} X_{n}(x)=0
$$

where $A_{n}, B_{n}$ and $C_{n}$ are constants that define the shape and amplitude of the beam vibration. They can be evaluated from the boundary conditions associated with each beam [36]. The constant $\kappa_{n}$ is the frequency parameter associated with each beam. Substituting Eq. (4) into Eq. (1) yields

$$
E I \sum_{n=1}^{\infty} Y_{n}(t) \frac{d^{4} X_{n}(x)}{d x^{4}}+C \sum_{n=1}^{\infty} \frac{d^{\beta} Y_{n}(t)}{d t^{\beta}} X_{n}(x)+\mu \sum_{n=1}^{\infty} \frac{d^{2} Y_{n}(t)}{d t^{2}} X_{n}(x)=P(x, t)
$$

Multiplying Eq. (7) by $X_{m}(x)$ and integrating over the domain $0 \leqslant x \leqslant L$ yields

$$
\begin{aligned}
& \sum_{n=1}^{\infty} Y_{n}(t) \int_{0}^{L} E I \frac{d^{4} X_{n}(x)}{d x^{4}} X_{m}(x) d x+\sum_{n=1}^{\infty} \frac{d^{\beta} Y_{n}(t)}{d t^{\beta}} \int_{0}^{L} C X_{n}(x) X_{m}(x) d x+ \\
& \sum_{n=1}^{\infty} \frac{d^{2} Y_{n}(t)}{d t^{2}} \int_{0}^{L} \mu X_{n}(x) X_{m}(x) d x=\int_{0}^{L} X_{m}(x) P(x, t) d x .
\end{aligned}
$$

Considering the orthogonality condition

$$
\int_{0}^{L} \mu X_{n}(x) X_{m}(x) d x=\left\{\begin{array}{lll}
0 & \text { if } & m \neq n \\
M_{n} & \text { if } & m=n
\end{array},\right.
$$

where $M_{n}$ is the generalized mass of the beam associated with the nth mode. In view of Eqs (6), (8) and (9), the differential equation of the nth mode of the generalized displacement or the modal response is written as

$$
\frac{d^{2} Y_{n}(t)}{d t^{2}}+2 \omega_{n} \zeta_{n} \frac{d^{\beta} Y_{n}(t)}{d t^{\beta}}+\omega_{n}^{2} Y_{n}(t)=\frac{Q_{n}(t)}{M_{n}}
$$


where

$$
\begin{aligned}
& \omega_{n}=\kappa_{n}^{2} \sqrt{E I / \mu}, \\
& \zeta_{n}=C / 2 \mu \omega_{n}, \\
& Q_{n}(t)=\int_{0}^{L} X_{n}(x) P(x, t) d x,
\end{aligned}
$$

and

$$
M_{n}=\int_{0}^{L} \mu X_{n}^{2}(x) d x
$$

are, respectively, the undamped natural circular frequency $\left(\omega_{n}\right)$, damping ratio $\left(\zeta_{n}\right)$, generalized load $\left(Q_{n}(t)\right)$ and the mass $\left(M_{n}\right)$ of beam associated with the nth mode. In Eqs (7) and (13), the load $P(x, t)$ which moves over beam from left to right is written as

$$
P(x, t)=P_{0} \delta[x-f(t)],
$$

where $P_{0}$ is the intensity of the applied moving load and $f(t)$ where $f(t)=v t$ is a function describing the motion of the force at time $\mathrm{t}$, in which $v$ being the constant speed of the moving load. Hence, Eq. (15) becomes

$$
P(x, t)=P_{0} \delta(x-v t) .
$$

Substituting Eq. (16) into Eq. (13) yields

$$
Q_{n}(t)=P_{0} \int_{0}^{L} X_{n}(x) \delta(x-v t) d x=P_{0} X_{n}(v t) .
$$

Substituting Eq. (17) into Eq. (10) yields

$$
\frac{d^{2} Y_{n}(t)}{d t^{2}}+2 \omega_{n} \zeta_{n} \frac{d^{\beta} Y_{n}(t)}{d t^{\beta}}+\omega_{n}^{2} Y_{n}(t)=\frac{P_{0}}{M_{n}} X_{n}(v t) .
$$

This equation satisfies the homogeneous initial conditions; $Y_{n}(0)=0$ and $\frac{d Y_{n}(0)}{d t}=0$. To find the exact solution of the second order initial value problem in Eq. (18), the solution $Y_{n}(t)$ can be decomposed into an infinite series of the form

$$
Y_{n}(t)=\sum_{k=0}^{\infty} Y_{n}^{k}(t)
$$

where $Y_{n}^{k}(t) ; k=0,1,2, \ldots$ are the components of $Y_{n}(t)$. It should be mentioned that the solution of Eq. (18) for the case of $\beta=1$ is extensively studied in the literature [3-20]. The components of $Y_{n}(t)$ can be determined recursively by substituting Eq. (19) into Eq. (18) as

$$
\begin{aligned}
& \frac{d^{2} Y_{n}^{0}(t)}{d t^{2}}+\omega_{n}^{2} Y_{n}^{0}(t)=\frac{P_{0}}{M_{n}} X_{n}(v t), \\
& \frac{d^{2} Y_{n}^{k+1}(t)}{d t^{2}}+\omega_{n}^{2} Y_{n}^{k+1}(t)=-2 \omega_{n} \zeta_{n} \frac{d^{\beta} Y_{n}^{k}(t)}{d t^{\beta}}, \quad k \geqslant 0 .
\end{aligned}
$$

Using the Laplace transform for the Caputo fractional derivative defined in Eq. (3) as

$$
L\left\{\frac{d^{\beta} Y_{n}^{k}(t)}{d t^{\beta}}\right\}=s^{\beta} y_{n}^{k}(s)-\sum_{j=0}^{m-1} s^{\beta-j-1}\left(y_{n}^{k}\right)^{j}(0), m-1<\beta \leqslant m .
$$

The Laplace transforms of Eqs (19) and (20), assuming homogeneous initial conditions can be written as 


$$
\begin{aligned}
& y_{n}(s)=\sum_{k=0}^{\infty} y_{n}^{k}(s), \\
& s^{2} y_{n}^{0}(s)+\omega_{n}^{2} y_{n}^{0}(s)=\frac{P_{0}}{M_{n}} L\left\{X_{n}(v t)\right\}=\frac{P_{0}}{M_{n}} \Phi(s), \\
& s^{2} y_{n}^{k+1}(s)+\omega_{n}^{2} y_{n}^{k+1}(s)=-2 \zeta_{n} \omega_{n} s^{\beta} y_{n}^{k}(s),
\end{aligned}
$$

where $L\{$.$\} designates the Laplace transform of \{$.$\} and L\left\{X_{n}(v t)\right\}=\Phi(s)$. Hence, the last two equations can be rearranged as

$$
\begin{aligned}
& y_{n}^{0}(s)=\frac{P_{0} \Phi(s)}{M_{n}\left(s^{2}+\omega_{n}^{2}\right)}, \\
& y_{n}^{k+1}(s)=\left(-2 \zeta_{n} \omega_{n}\right)^{k+1} \frac{P_{0} s^{(k+1) \beta} \Phi(s)}{M_{n}\left(s^{2}+\omega_{n}^{2}\right)^{k+2}}, k=0,1,2, \ldots
\end{aligned}
$$

Therefore Eq. (22) becomes

$$
\begin{aligned}
y_{n}(s) & =\frac{P_{0} \Phi(s)}{M_{n}} \sum_{k=0}^{\infty}(-1)^{k}\left(2 \zeta_{n} \omega_{n}\right)^{k} \frac{s^{\beta k}}{\left(s^{2}+\omega_{n}^{2}\right)^{k+1}} \\
& =\frac{P_{0} \Phi(s)}{M_{n}\left(s^{2}+\omega_{n}^{2}\right)}+\frac{P_{0} \Phi(s)}{M_{n}} \sum_{k=1}^{\infty}(-1)^{k}\left(2 \zeta_{n} \omega_{n}\right)^{k} \frac{s^{\beta k}}{\left(s^{2}+\omega_{n}^{2}\right)^{k+1}} .
\end{aligned}
$$

The term by term inversion of the series presented in Eq. (26), based on the general expansion theorem for the Laplace transform, is written as [33]

$$
\begin{aligned}
G(t) & =\sum_{k=1}^{\infty} \frac{(-1)^{k}}{k !}\left(2 \zeta_{n} \omega_{n}\right)^{k} t^{2 k-\beta k+1} E_{2,2-\beta k}^{(k)}\left(-\omega_{n}^{2} t^{2}\right) \\
& =\sum_{j=0}^{\infty} \sum_{k=1}^{\infty} \frac{(-1)^{k+j}\left(2 \zeta_{n} \omega_{n}\right)^{k} \Gamma(j+k+1) \omega_{n}^{2 j} t^{(2 j+2 k-\beta k+1)}}{\Gamma(k+1) \Gamma(j+1) \Gamma(2 j+2 k-\beta k+2)},
\end{aligned}
$$

where $k !=\Gamma(k+1)$ and $E_{\lambda, \mu}(y)$ is the Mittag-Leffler function in two parameters, defined as [33]

$$
E_{\lambda, \mu}^{(k)}(y) \equiv \frac{d^{k}}{d y^{k}} E_{\lambda, \mu}(y)=\sum_{j=0}^{\infty} \frac{(j+k) ! y^{j}}{j ! \Gamma(\lambda j+\lambda k+\mu)} \quad(k=0,1,2, \ldots),
$$

and

$$
L^{-1}\left\{\frac{s^{\lambda-\mu}}{\left(s^{\lambda} \mp a\right)^{k+1}}\right\}=\frac{t^{\lambda k+\mu-1} E_{\lambda, \mu}^{(k)}( \pm a t)}{k !}, \quad \Re e(s)>|a|^{1 / \lambda}
$$

In view of the last two equations, taking the inverse Laplace transform of Eq. (26) and using the convolution theorem we, get

$$
Y_{n}(t)=\frac{P_{0}}{M_{n} \eta_{1}}\left[\eta_{2}+\eta_{3}\right]+\sum_{j=0}^{\infty}(-1)^{j}\left(\omega_{n}^{2}\right)^{j}\left[\eta_{4}\left\{\eta_{5}-\eta_{6}+\eta_{7}+\eta_{8}\right\}\right]
$$

\section{where}

$$
\begin{aligned}
& \eta_{1}=\omega_{n}^{2}+\Omega_{n}^{2} \\
& \eta_{2}=\left[C_{n} \cosh \left(\Omega_{n} t\right)-C_{n} \cos \left(\omega_{n} t\right)+B_{n} \sinh \left(\Omega_{n} t\right)-\frac{B_{n} \Omega_{n} \sin \left(\omega_{n} t\right)}{\omega_{n}}\right]
\end{aligned}
$$




$$
\begin{aligned}
& \eta_{3}=\left[\frac{\eta_{1}}{\omega_{n}^{2}-\Omega_{n}^{2}}\left[A_{n} \cos \left(\Omega_{n} t\right)-A_{n} \cos \left(\omega_{n} t\right)+\sin \left(\Omega_{n} t\right)-\frac{\Omega_{n} \sin \left(\omega_{n} t\right)}{\omega_{n}}\right]\right] \\
& \eta_{4}=\left[\sum_{k=1}^{\infty}\left(\frac{\Gamma(j+k+1)\left(-2 \omega_{n} \xi_{n}\right)^{k} P_{0}}{M_{n} \Gamma(j+1) \Gamma(k+1) \Gamma(\gamma+2)}\right)\right] \\
& \eta_{5}=\left[\left\{\frac{\Omega_{n}^{(-5 / 2-\gamma)}\left(\Omega_{n} t+A_{n}\right) \text { LommelS } 1\left(\frac{3}{2}+\gamma, \frac{1}{2}, \Omega_{n} t\right)}{\sqrt{t}}\right\}\right] \\
& \eta_{6}=\left[\left\{\sqrt{t} A_{n} \Omega_{n}^{(-3 / 2-\gamma)}(-\gamma) \text { LommelS } 1\left(\frac{1}{2}+\gamma, \frac{3}{2}, \Omega_{n} t\right)\right\}\right] \\
& \eta_{7}=\left[\left\{\frac{B_{n} \Omega_{n} t^{(\gamma+3)} \text { hypergeom }\left([1],\left[\frac{5}{2}+\frac{\gamma}{2}, 2+\frac{\gamma}{2}\right], \frac{1}{4} \Omega_{n}^{2} t^{2}\right)}{(\gamma+3)(\gamma+2)}\right\}\right] \\
& \eta_{8}=\left[\left\{\frac{C_{n} t^{(\gamma+2)} \text { hypergeom }\left([1],\left[\frac{3}{2}+\frac{\gamma}{2}, 2+\frac{\gamma}{2}\right], \frac{1}{4} \Omega_{n}^{2} t^{2}\right)}{(2 j+2 k-k \beta+2)}\right\}\right]
\end{aligned}
$$

$\gamma=2 j+2 k-k \beta$ and $\Omega_{n}=\kappa_{n} v$

In Eq. (31) hypergeom(.) and LommelS1(.) are, respectively, the generalized hyper-geometric and Lommel functions defined as [37]:

$$
\begin{aligned}
& \text { hypergeom }\left(\left[n_{1}\right],\left[d_{1}, d_{2}\right], z\right)=\sum_{k=0}^{\infty} \frac{z^{k}\left(\frac{\Gamma\left(n_{1}+k\right)}{\Gamma\left(n_{1}\right)}\right)}{k !\left(\prod_{j=1}^{2} \frac{\Gamma\left(d_{j}+k\right)}{\Gamma\left(d_{j}\right)}\right)} \\
& \operatorname{Lommel} S 1(a, b, z)=\frac{z^{a+1} \text { hypergeom }\left([1],\left[\frac{3}{2}-\frac{1}{2} b+\frac{1}{2} a, \frac{3}{2}+\frac{1}{2} b+\frac{1}{2} a\right],-\frac{1}{4} z^{2}\right)}{(a-b+1)(a+b+1)}
\end{aligned}
$$

Hence, the general transverse deflection of the beam can be found by substituting Eq. (5) and Eqs (30-32) into Eq. (4).

In the aforementioned formulation and by introducing the Mittag-Leffler function in two parameters, the method ended with infinite series solution. The second term of this solution, Eq. (30), i.e. the infinite series term, becomes zero as the generalized damping ratio of the nth mode $\zeta_{n}$ goes to zero. However, the remaining non-series part fit exactly the analytical solution for undamped beams obtained by the Duhamel integral. For example, for a simply supported undamped beam $\left(A_{n}=B_{n}=C_{n}=\zeta_{n}=0\right)$, Eq. (30) reduces to

$$
Y_{n}(t)=\frac{P_{0}}{M_{n}\left(\omega_{n}^{2}-\Omega_{n}^{2}\right)}\left[\sin \left(\Omega_{n} t\right)-\frac{\Omega_{n} \sin \left(\omega_{n} t\right)}{\omega_{n}}\right],
$$

This precisely fits the exact solution obtained by the Duhamel integral [4].

\section{Results and discussion}

Two verification problems are given below to verify the validity of the technique proposed in this paper. The dynamic response of fractionally damped beams with various orders of fractional derivatives is also discussed in this section. 

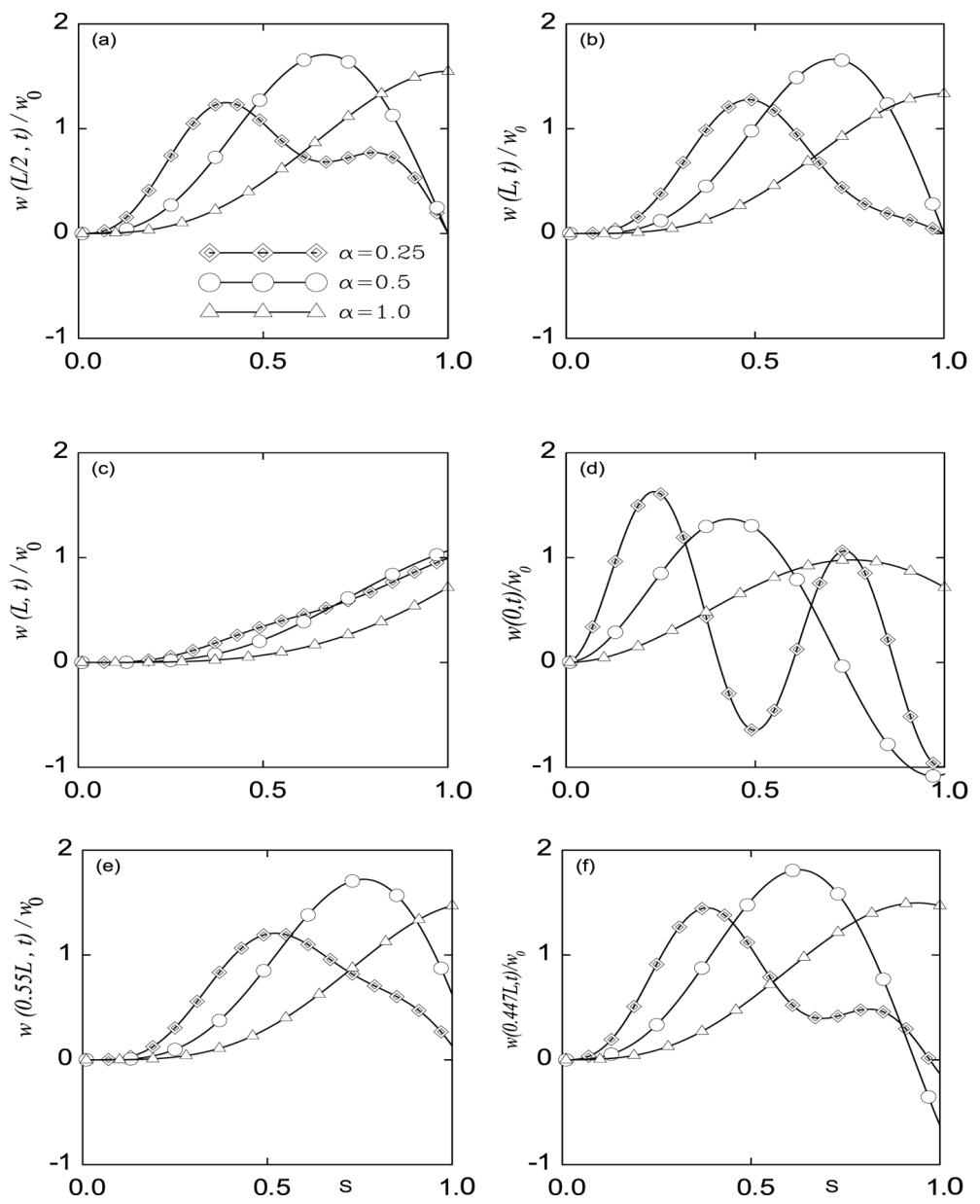

Fig. 1. Dynamic response $\left(w\left(x_{\max }, t\right) / w_{0}\right)$ for various values of $\alpha$ and $\zeta_{n}=0$ at $x_{\max }$ for (a) hinged-hinged, (b) fixed-fixed, (c) fixed-free, (d) free-fixed, (e) fixed-hinged, and (f) hinged-fixed beams.

\subsection{Verification problem 1}

In this problem the response of an initially stationary oscillator whose damping is prescribed by a fractional derivative of order $\beta=0.5$ will be examined. The oscillator is assumed to be subjected to an excitation of the form $P(x, t)=P_{0} H(t)$, where $H(t)$ is the Heaviside unit step function and $P_{0}$ is the intensity of the load. Hence, this is a single-degree-of-freedom spring-mass-damper system whose dynamic response can be prescribed by the following differential equation [30]

$$
\frac{d^{2} x(t)}{d t^{2}}+\frac{c}{m} \frac{d^{1 / 2} x(t)}{d t^{1 / 2}}+\frac{k}{m} x(t)=\frac{P_{0} H(t)}{m}
$$

where $m, c$ and $k$ represent the mass, damping coefficient, and stiffness coefficient of the oscillator, respectively. Following the procedure of Eqs (19) to (26), the solution of Eq. (34) in the Laplace domain can be written directly as

$$
X(s)=\frac{P_{0}}{m} \sum_{k=0}^{\infty}(-1)^{k}\left(\frac{c}{m}\right)^{k} \frac{s^{\beta k-1}}{\left(s^{2}+\omega^{2}\right)^{k+1}},
$$

where $L\{x(t)\}=X(s)$ and $\omega=\sqrt{k / m}$. In view of Eq. (29), the term by term inversion of the series presented in Eq. (35) leads to 
Table 1

Maximum static deflection and its corresponding location of the studied beams

\begin{tabular}{lcccccc}
\hline & Hinged-hinged & Fixed-fixed & Fixed-free & Free-fixed & Fixed-hinged & Hinged-fixed \\
\hline$w_{0}$ & $\frac{P_{0} L^{3}}{48 E I}$ & $\frac{P_{0} L^{3}}{192 E I}$ & $\frac{P_{0} L^{3}}{3 E I}$ & $\frac{P_{0} L^{3}}{3 E I}$ & $\frac{P_{0} L^{3}}{48 \sqrt{5} E I}$ & $\frac{P_{0} L^{3}}{48 \sqrt{5} E I}$ \\
\multirow{2}{*}{$x_{\max }$} & $\frac{L}{2}$ & $\frac{L}{2}$ & $L$ & 0 & $L\left(1-\frac{1}{\sqrt{5}}\right)$ & $\frac{L}{\sqrt{5}}$ \\
\hline
\end{tabular}
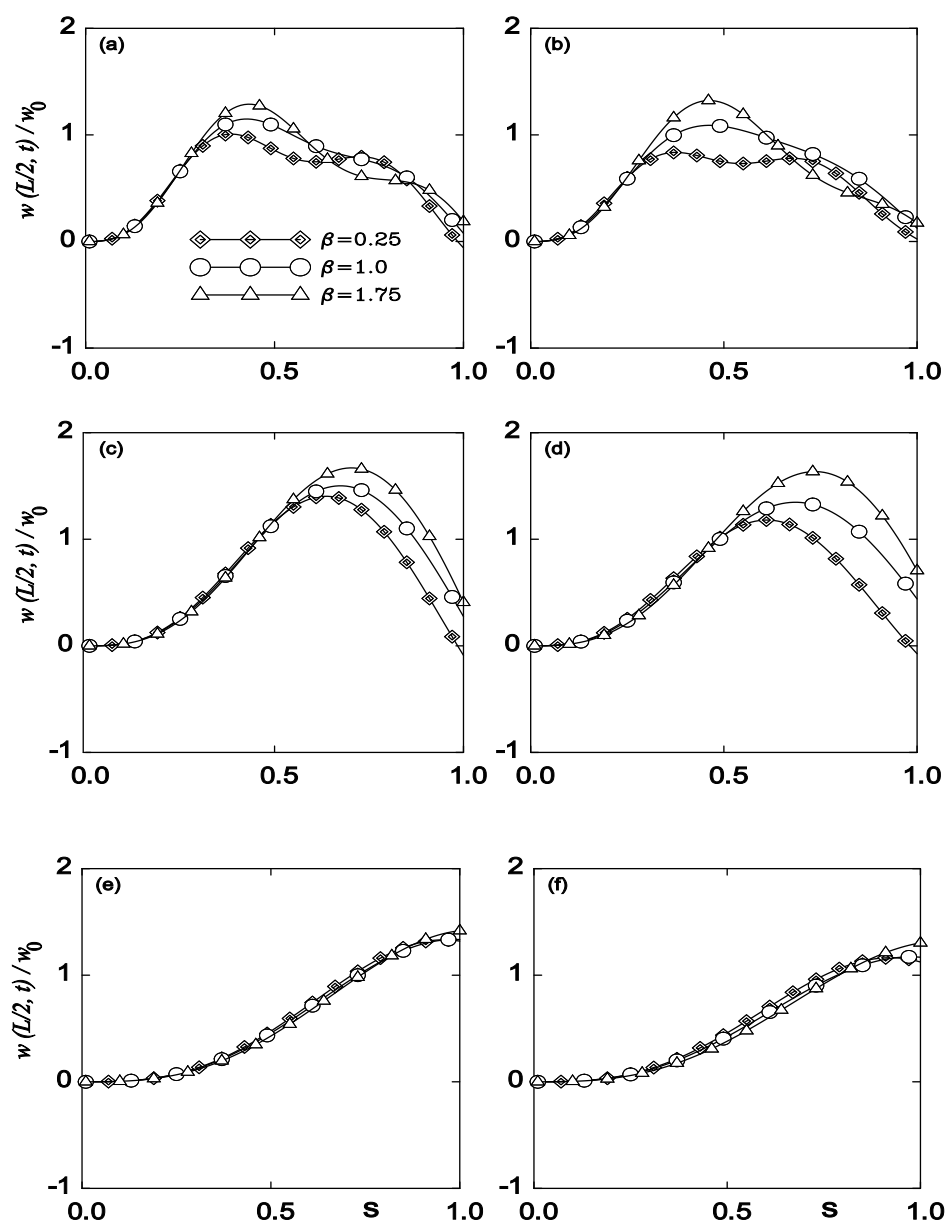

Fig. 2. Dynamic response $\left(w\left(\frac{L}{2}, t\right) / w_{0}\right)$ for a hinged-hinged beam: (a) $\alpha=0.25, \zeta_{n}=0.1$, (b) $\alpha=0.25, \zeta_{n}=0.2$, (c) $\alpha=0.5, \zeta_{n}=0.1$, (d) $\alpha=0.5, \zeta_{n}=0.2$, (e) $\alpha=1, \zeta_{n}=0.1$, (f) $\alpha=1, \zeta_{n}=0.2$.

$$
\begin{aligned}
x(t) & =\frac{P_{o}}{m} \sum_{k=0}^{\infty} \frac{(-1)^{k}}{k !}\left(\frac{c}{m}\right)^{k} t^{2 k-\beta k+2} E_{2,3-\beta k}^{(k)}\left(-\omega^{2} t^{2}\right) \\
& =\sum_{j=0}^{\infty} \sum_{k=0}^{\infty} \frac{(-1)^{k+j}\left(\frac{c}{m}\right)^{k} \Gamma(j+k+1) \omega^{2 j} t^{(2 j+2 k-\beta k+2)}}{\Gamma(k+1) \Gamma(j+1) \Gamma(2 j+2 k-\beta k+3)},
\end{aligned}
$$

This can be written for $\beta=0.5$ as

$$
x(t)=\sum_{j=0}^{\infty} \sum_{k=0}^{\infty} \frac{(-1)^{k+j}\left(\frac{c}{m}\right)^{k} \Gamma(j+k+1) \omega^{2 j} t^{\left(2 j+\frac{3}{2} k+2\right)}}{\Gamma(k+1) \Gamma(j+1) \Gamma\left(2 j+\frac{3}{2} k+3\right)} .
$$



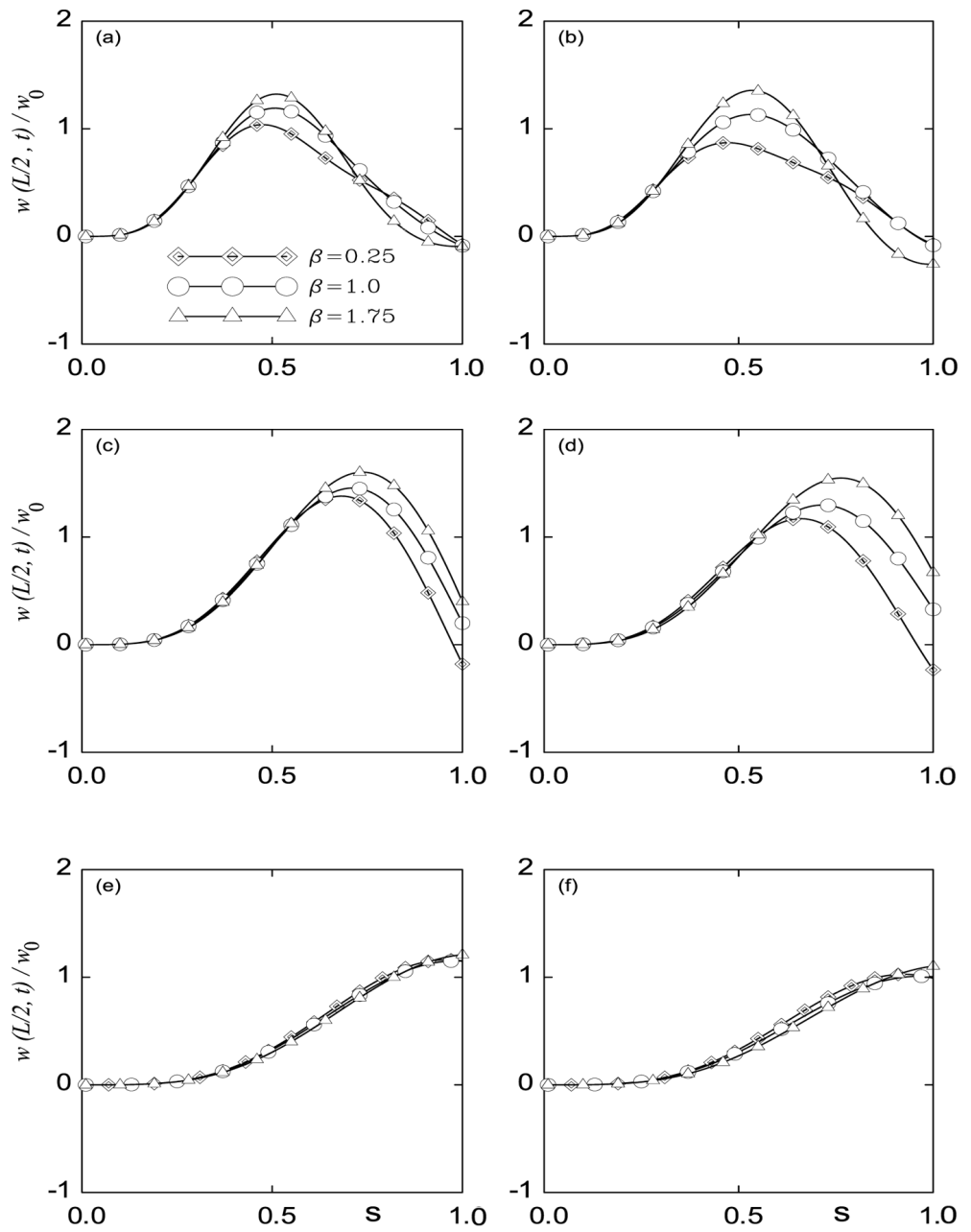

Fig. 3. Dynamic response $\left(w\left(\frac{L}{2}, t\right) / w_{0}\right)$ for a fixed-fixed beam: (a) $\alpha=0.25, \zeta_{n}=0.1$, (b) $\alpha=0.25, \zeta_{n}=0.2$, (c) $\alpha=0.5, \zeta_{n}=0.1$, (d) $\alpha=0.5, \zeta_{n}=0.2$, (e) $\alpha=1, \zeta_{n}=0.1$, (f) $\alpha=1, \zeta_{n}=0.2$.

This solution is exactly the same as that presented in [30], which is derived using the Adomian decomposition method. Furthermore, the solution presented in Eq. (37) fits exactly the fractional Green's functions solution discussed by Podlubny [33] for the fractional differential equation presented in Eq. (34).

\subsection{Verification problem 2}

The well known inhomogeneous Bagley-Torvik initial value problem is investigated. The mathematical model of this problem is given as $[33,38]$

$$
\frac{d^{2} y(t)}{d t^{2}}+\frac{B}{A} \frac{d^{3 / 2} y(t)}{d t^{3 / 2}}+\frac{C}{A} y(t)=\frac{f(t)}{A}, t>0
$$

where $y(0)=0, \frac{d y(0)}{d t}=0, A \neq 0$. Moreover, in Eq. (38) $A, B$ and $C$ are constants. Following the same steps of verification used in problem 1 , the analytical solution of Eq. (38) can be expressed as

$$
y(t)=L^{-1}\left(\frac{F(s)}{A} \sum_{k=0}^{\infty}(-1)^{k}\left(\frac{B}{A}\right)^{k} \frac{s^{\frac{3 k}{2}}}{\left(s^{2}+\frac{C}{A}\right)^{k+1}}\right)
$$



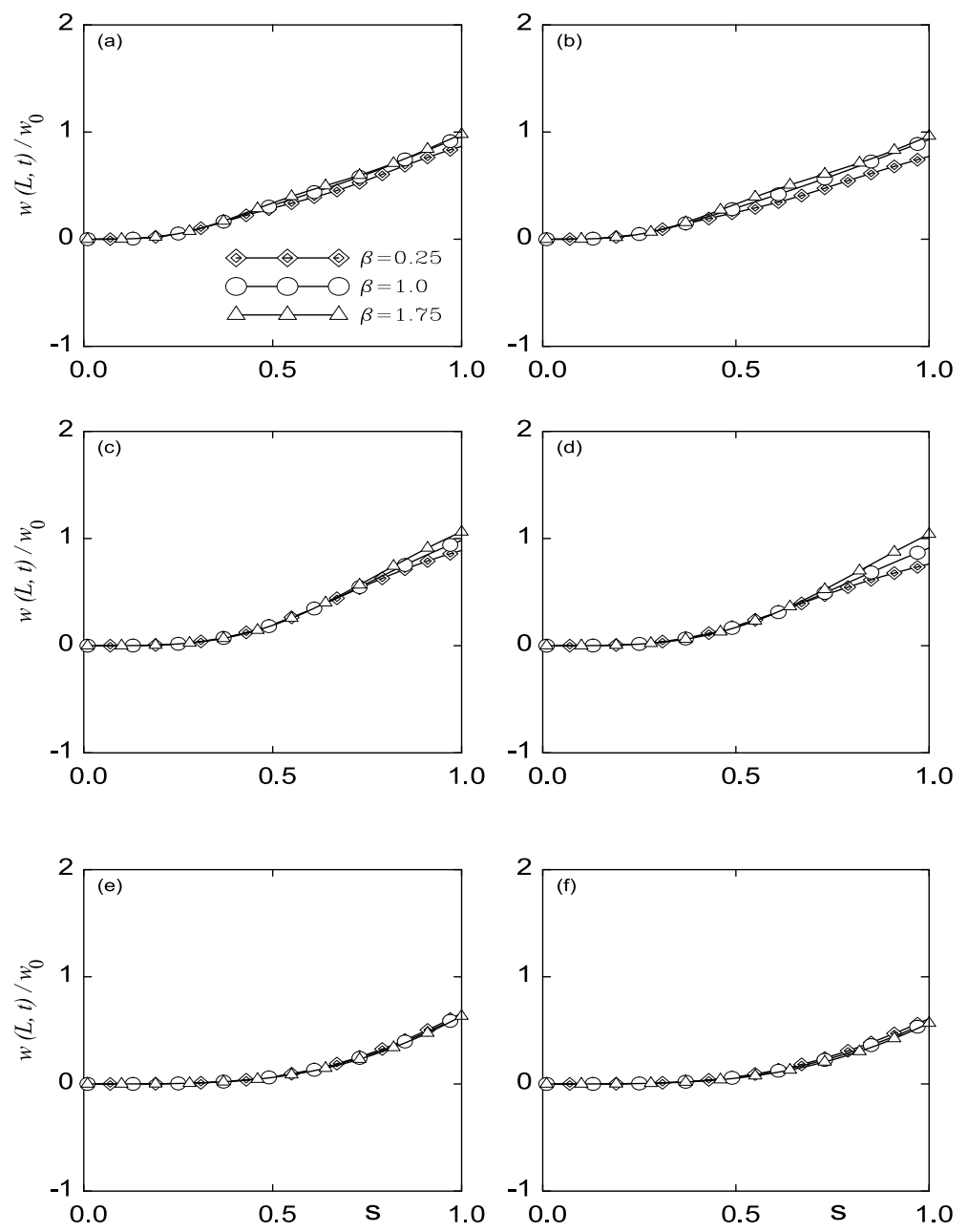

Fig. 4. Dynamic response $\left(w(L, t) / w_{0}\right)$ for a fixed-free beam: (a) $\alpha=0.25, \zeta_{n}=0.1$, (b) $\alpha=0.25, \zeta_{n}=0.2$, (c) $\alpha=0.5, \zeta_{n}=0.1$, (d) $\alpha=$ $0.5, \zeta_{n}=0.2$, (e) $\alpha=1, \zeta_{n}=0.1$, (f) $\alpha=1, \zeta_{n}=0.2$.

$$
=\int_{0}^{t}\left(\frac{f(\tau)}{A}\right)\left(\sum_{j=0}^{\infty} \sum_{k=0}^{\infty} \frac{(-1)^{k+j}\left(\frac{B}{A}\right)^{k} \Gamma(j+k+1)\left(\frac{C}{A}\right)^{j}(t-\tau)^{\left(2 j+\frac{1}{2} k+1\right)}}{\Gamma(k+1) \Gamma(j+1) \Gamma\left(2 j+\frac{1}{2} k+2\right)}\right) d \tau
$$

The solution presented in Eq. (39) agrees exactly with the fractional Green's functions solution discussed by Podlubny [33].

\subsection{Examples of beams with fractional derivative of various orders}

The analysis outcomes from this paper are applied to homogeneous isotropic fractionally damped beams with the four classical boundary conditions with six combinations. Hinged-hinged, fixed-fixed, fixed-free, free-fixed, fixed-hinged, and hinged-fixed beams are used to clarify the results. The beams are subjected to concentrated constant loads moving with uniform velocity. At this point, it is worth mentioning that some special cases (related to $\beta=1$ ) perfectly fit the results presented in reference $[3,18]$. In here, the beams are assumed to be at rest when a concentrated force $P_{0}$ enters the beam from the left-hand side. In Figs 1-7, the dimensionless dynamic deflection

$$
\bar{w}(x, t)=\frac{w\left(x_{\max }, t\right)}{w_{0}},
$$



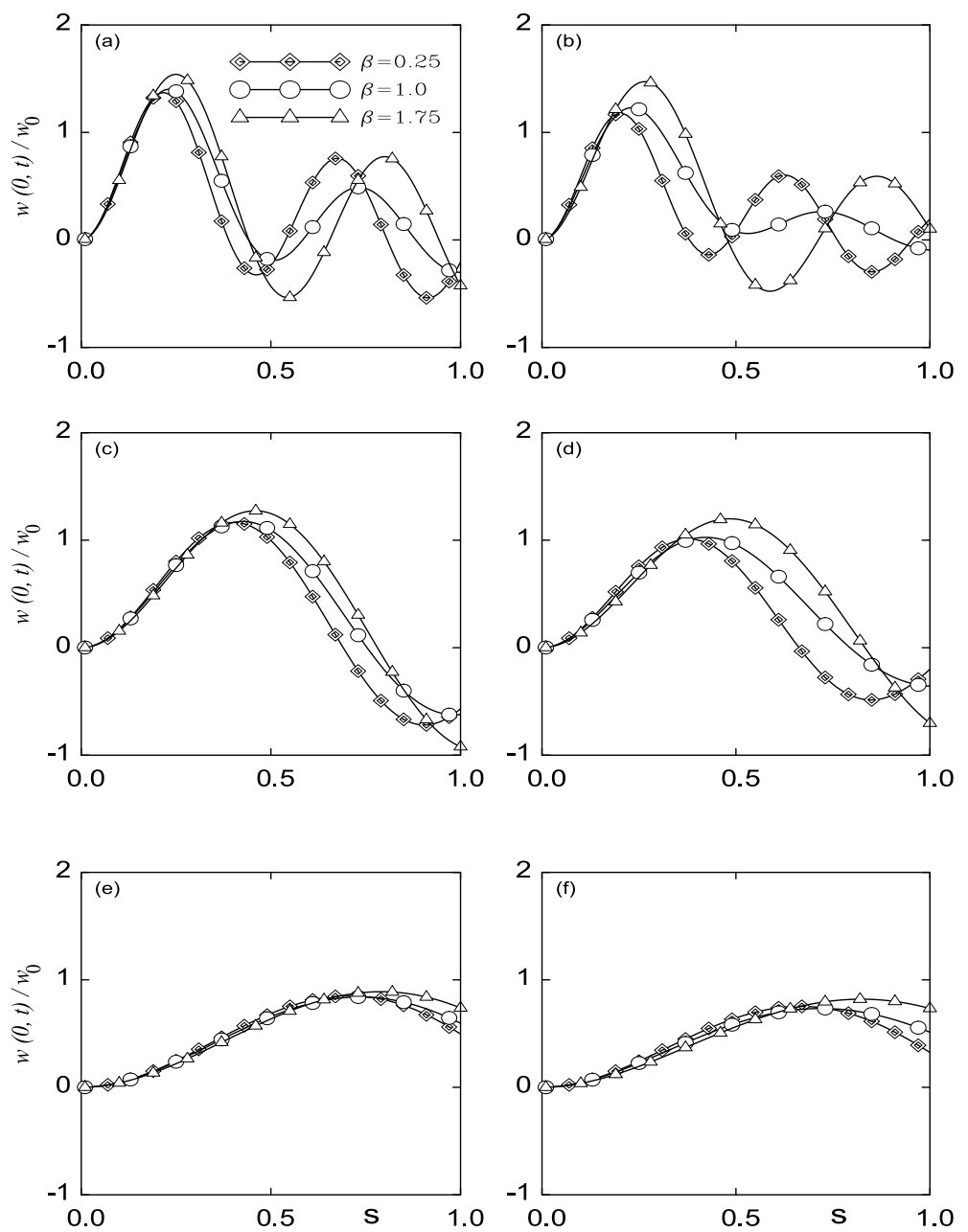

Fig. 5. Dynamic response $\left(w(0, t) / w_{0}\right)$ for a free-fixed beam: (a) $\alpha=0.25, \zeta_{n}=0.1$, (b) $\alpha=0.25, \zeta_{n}=0.2$, (c) $\alpha=0.5, \zeta_{n}=0.1$, (d) $\alpha=$ $0.5, \zeta_{n}=0.2$, (e) $\alpha=1, \zeta_{n}=0.1$, (f) $\alpha=1, \zeta_{n}=0.2$.

is shown for all beams versus the dimensionless time parameter $s=\frac{t}{t_{f}}=\frac{t v}{L}$, where $t_{f}=L / v$ is the instant at which the force arrives the right-hand side of the beam. Thus, when $s=0$, the force is at the left-hand side of the beam, and when $s=1$, the force is at the right-hand side of the beam. In Eq. (40) as well as in the following discussion $w_{0}$ and $x_{\max }$ denotes, respectively, the maximum static deflection and the point on the beam corresponds to maximum deflection, Table 1 [38]. The deflection $w\left(x_{\max }, t\right)$ is obtained from substituting Eqs (30-32) and (5) into (4). In Figs 1-7, the effects of damping, speed of the load and the order of the fractional derivative are clearly presented. The effect of speed is represented by the dimensionless speed parameter $\alpha$ which is defined as

$$
\alpha=v / c_{c r},
$$

where $c_{c r}$ is the critical speed, defined as $[3,4]$

$$
c_{c r}=\omega_{1} L / \pi .
$$

Results presented in Figs 1-7 are obtained for three values of $\alpha$ : 0.25, 0.5 and 1, three values of the fractional derivative $\beta: 0.25,1$ and 1.75, and three values of the damping coefficient $\zeta_{n}: 0,0.1$ and 0.2 . Figure 1 (a-f), shows the dimensionless dynamic deflection $\left(w\left(x_{\max }, t\right) / w_{0}\right)$ versus the dimensionless time $(s)$ for the six beams under consideration for the values of $w_{0}$ and $x_{\max }$ presented in Table 1. The curves in Fig. $1\left(\zeta_{n}=0\right)$ fit exactly the corresponding ones presented in $[3,18]$. These curves show that the dynamic deflection of the fixed-fixed beam, 

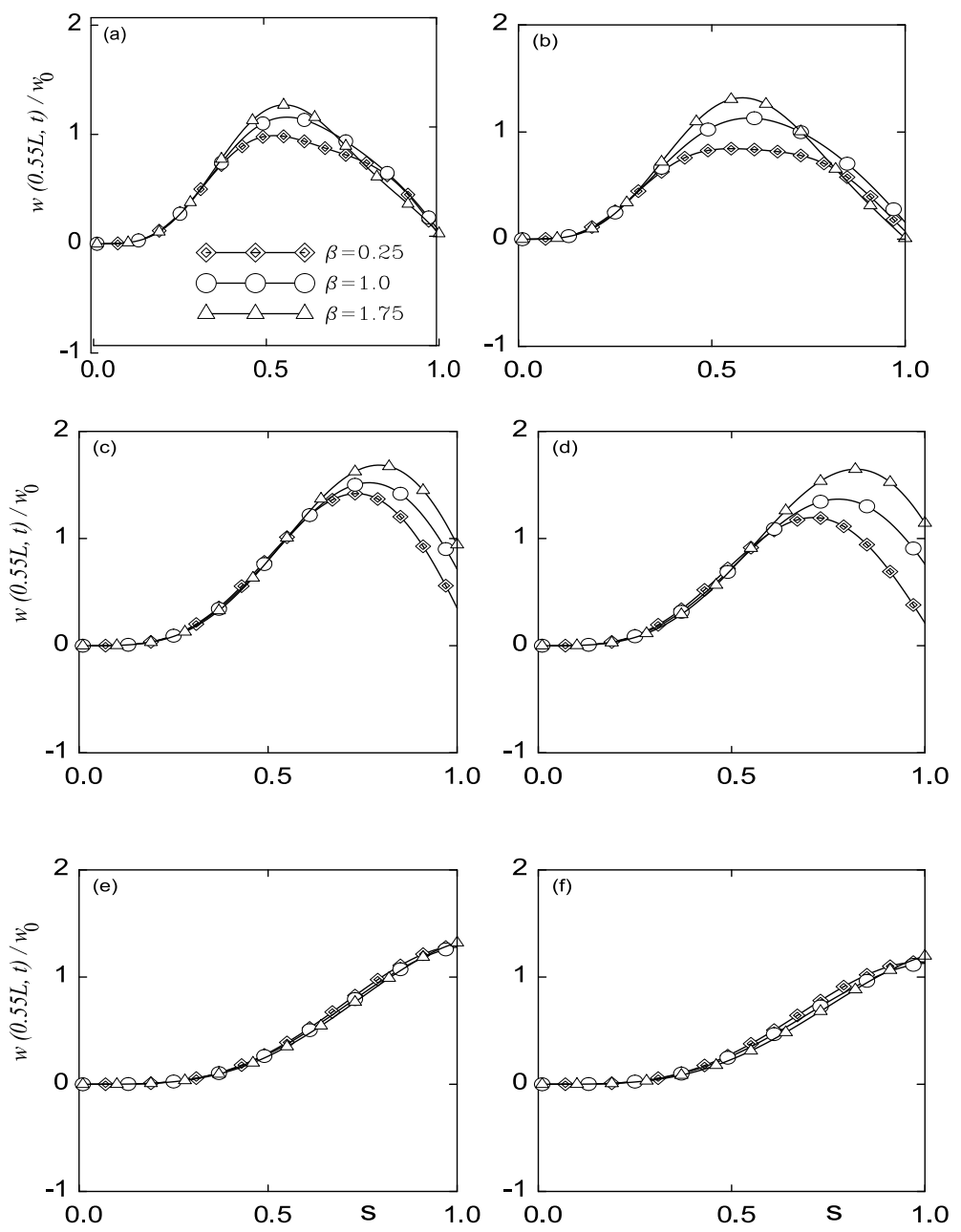

Fig. 6. Dynamic response $\left(w(0.55 L, t) / w_{0}\right)$ for a fixed-hinged beam: (a) $\alpha=0.25, \zeta_{n}=0.1$, (b) $\alpha=0.25, \zeta_{n}=0.2$, (c) $\alpha=0.5, \zeta_{n}=0.1$, (d) $\alpha=0.5, \zeta_{n}=0.2$, (e) $\alpha=1, \zeta_{n}=0.1$, (f) $\alpha=1, \zeta_{n}=0.2$.

Fig. 1.b, is smaller than that of the simply supported beam, Fig. 1.a. This is due to the difference in boundary conditions at the ends. Furthermore, the difference between the dimensionless deflection for the fixed-free, Fig. 1.c, and free-fixed, Fig. 1.d, beams when the speed parameter is small is due to entrance point of the load, i.e., for the fixed-free beam the load enters the beam from the fixed end while for the free-fixed beam it enters from the free end. Furthermore, the maximum value of the dynamic deflection of the fixed-free beam is less than that corresponds to the free-fixed beam at the free end. Moreover, the free end oscillates more when the load enters the beam from the free end. Results also show that the dynamic deflection of the fixed-hinged beam, Fig. 1.e, is smaller than that of the hinged-fixed, Fig. 1.f, because the load enters the beam from the fixed end for the fixed-hinged beam. From Fig. 1, it can be noted that the free-fixed beam, Fig. 1.d, suffer more oscillations than other beams because it is the only case where the load enters the beam from the free end. Similar trends can also be observed from Figs 3-7. Figure $2(\mathrm{a}-\mathrm{f})$, shows the dimensionless dynamic deflection $\left(w\left(x_{\max }, t\right) / w_{0}\right)$ versus the dimensionless time $(s)$ for a hinged-hinged beam at $x_{\max }=L / 2$ for $w_{0}=\frac{P_{0} L^{3}}{48 E I}$. Figure $2(\mathrm{a}, \mathrm{b}),(\mathrm{c}, \mathrm{d})$ and $(\mathrm{e}, \mathrm{f})$ is drawn, respectively, for $\alpha=$ $0.25,0.5$ and 1, however, Fig. 2 (a, c, e) and (b, d, f) is drawn for $\zeta_{n}=0.1$ and 0.2 , respectively. In these figures, the effect of damping is shown clearly, where the increase in damping coefficient yields, in general, a decrease in the response. From the curves of Fig. 2, it is further noted that as the order of the fractional derivative $(\beta)$ increases the amplitude of the response increases, Fig. 2 (a-d). Furthermore, these curves reveal that as the order of the fractional derivative $(\beta)$ increases the peak of the dynamic deflection shifts to the right, this means that the smaller the value of 

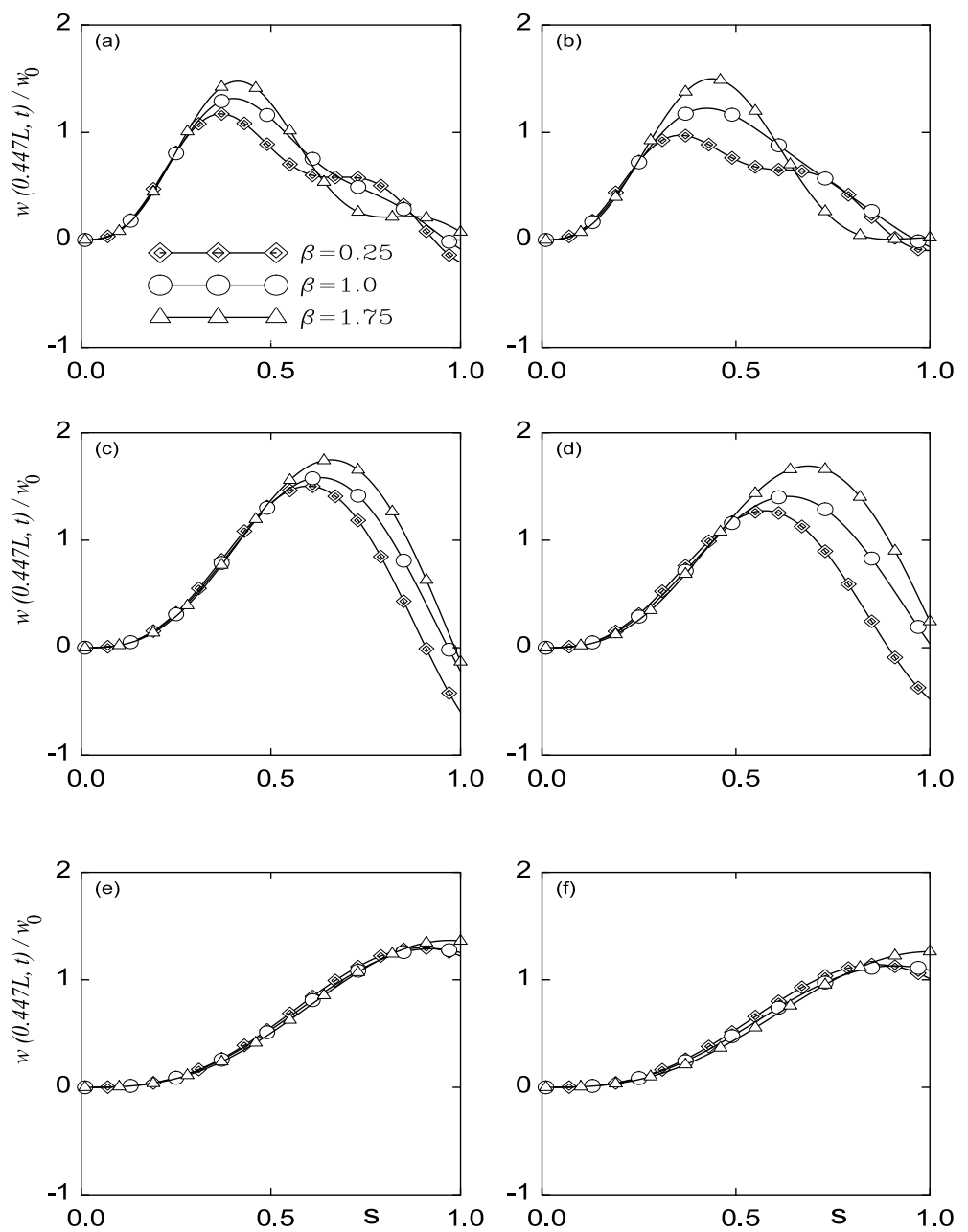

Fig. 7. Dynamic response $\left(w(0.447 L, t) / w_{0}\right)$ for a hinged-fixed beam: (a) $\alpha=0.25, \zeta_{n}=0.1$, (b) $\alpha=0.25, \zeta_{n}=0.2,(\mathrm{c}) \alpha=0.5, \zeta_{n}=$ 0.1, (d) $\alpha=0.5, \zeta_{n}=0.2$, (e) $\alpha=1, \zeta_{n}=0.1$, (f) $\alpha=1, \zeta_{n}=0.2$.

the derivative order $\beta$, the more oscillations the beams suffer, Fig. 2 (a-d). A very good agreement is found between the curves of Figs 2-7 for $\beta=1$ and the corresponding curves presented in [18]. In a similar manner, Figs 3-7 revealed that the dynamic response decreases as the damping factor $\zeta_{n}$ increases. From the curves of Figs 2-7, it is further noted that as the order of the fractional derivative $(\beta)$ increases the maximum amplitude of the response increases. Furthermore, in general these curves reveal that as the order of the fractional derivative $(\beta)$ increases the peak of the dynamic deflection shifts to the right, this means that smaller the value of the derivative order $\beta$, the more oscillations the beams suffer. Hence, the effect of the order of fractional derivative becomes clear.

\section{Conclusions and recommendations}

An analytical model is presented to solve transverse vibration of Bernoulli-Euler beams with damping characteristics that are described in terms of fractional derivatives of arbitrary order. The method employed is the Laplace transform with the decomposition method to find the analytical solution. Numerical results presented in this paper, show that: 1) the dynamic response increases as the damping factor decreases, 2) the smaller value of the derivative order $\beta$ is, the more oscillations the beams suffer and 3) the dynamic response increases as the fractional derivative order increases. 
Although the method used has the ability to get the infinite series solution directly by introducing the MittagLeffler function. This solution may be written in a closed form for some special cases; otherwise, it may be truncated and fulfilled easily by using mathematical soft ware like Maple.

Finally, even though the fractional derivative provides better damping models of fractionally damped structures and materials, few papers have been presented in this field. This is probably due to the fact that the underlying mathematics of the fractional derivative models is not well developed. Hence, recent development in this field leads to the development of fractional derivative models in many engineering problems. The approach of the fractional derivative damping may allow researchers to choose suitable mathematical models that will precisely fit experimental models.

\section{Acknowledgment}

The authors would like to thank Prof. Dr. Yehya N. Abdellatif for reviewing this paper and providing his valuable corrections.

\section{References}

[1] G. Adomian, A new approach to nonlinear partial differential equations, J Math Appl 102 (1984), $420-434$.

[2] G. Adomian, Solving Frontier Problems of Physics: The Decomposition Method, Kluwer Academic Publication, 1994.

[3] L. Fryba, Vibration of Solids and Structures under Moving Loads, Noordhoff International, Groningen, 1972.

[4] L. Fryba, Non-stationary response of a beam to a moving random force, Journal of Sound and Vibration 46 (1976), 323-338.

[5] P. Sniady, Dynamic response of linear structures to a random stream of pulses, Journal of Sound and Vibration 131 (1989), 91-102.

[6] H.S. Zibdeh, Stochastic vibration of an elastic beam due to random moving loads and deterministic axial forces, Engineering Structures 17 (1995), 530-535.

[7] H. S. Zibdeh and R. Rackwitz, Response moments of an elastic beam subjected to Poissonian moving loads, Journal of Sound and Vibration 188 (1995), 479-495.

[8] E. Savin, Dynamic amplification factor and response spectrum for the evaluation of vibrations of beams under successive moving loads, Journal of Sound and Vibration 248 (2001), 267-288.

[9] Y.B. Yang and C.W. Lin, Vehicle-bidge interaction dynamics and Potential applications, Journal of Sound and Vibration 284 (2005), 205-226.

[10] H.S. Zibdeh and H.S. Juma, Dynamic response of a rotating beam subjected to a random moving load, Journal of Sound and Vibration 223 (1999), 741-758.

[11] R. Katz, C.W. Lee, A.G. Ulsoy and R.A. Scott, The dynamic response of a rotating shaft subject to a moving load, Journal of Sound and Vibration 122 (1988), 131-148.

[12] A. Argento, H.L. Morano and R.A. Scott, Accelerating load on a rotating beam, Journal of Sound and Vibration 116 (1994), $397-403$.

[13] D. Thambiratnam and Y. Zhuge, Dynamic analysis of beams on an elastic foundation subjected to moving loads, Journal of Sound and Vibration 198 (1996), 149-169.

[14] A.K. Mallik, S. Chandra and A.B. Singh, Steady-state response of an elastically supported infinite beam to a moving load, Journal of Sound and Vibration 291 (2006), 1148-1169.

[15] M.A. Foda and Z. Abduljabbar, A dynamic Green function formulation for the response of a beam structure to a moving mass, Journal of Sound and Vibration 210 (1998), 295-306.

[16] G. Michaltsos, D. Sophianopoulos and A.N. Kounadis, The effect of a moving mass and other parameters on the dynamic response of a simply supported beam, Journal of Sound and Vibration 191 (1996), 357-362.

[17] B. Yang, C.A. Tan and L.A. Bergman, Direct numerical procedure for solution of moving oscillator problems, ASCE Journal of Engineering Mechanics 126 (2000), 462-469.

[18] M. Abu Hilal and H.S. Zibdeh, Vibration analysis of beams with general boundary conditions traversed by a moving force, Journal of Sound and Vibration 229 (2) (2000), 377-388.

[19] H.S. Zibdeh and R. Rackwitz, Moving loads on beams with general boundary conditions, Journal of Sound and Vibration 195(1) (1996), 85-102.

[20] T. Pritz, Five-parameter fractional derivative model for polymeric damping materials, Journal of Sound and Vibration 265(5) (2003), 935-952.

[21] H. Qi and M. Xu, Unsteady flow of viscoelastic fluid with fractional Maxwell model in a channel, Mechanics Research Communications 34(2) (2007), 210-212.

[22] R.L. Bagley and P.J. Torvik, On the fractional calculus model of viscoelastic behavior, Journal of Rheology 30 (1986), $133-135$.

[23] N. Shimizu and W. Zhang, Fractional calculus approach to dynamic problems of viscoelastic materials, JSME International Journal, Series C 42 (1999), 825-837.

[24] N. Makris and M.C. Constantinou, Fractional derivative Maxwell model for viscous damper, Journal of Structural Engineering, ASCE 117 (1991), 2708-2724. 
[25] C.G. Koh and J.M. Kelly, Application of fractional derivatives to seismic analysis of base-isolated models, Earthquake Engineering and Structural Dynamics 19 (1990), 229-241.

[26] K.S. Fa, A falling body problem through the air in view of the fractional derivative approach, Physica A 350 (2005), $199-206$.

[27] A. Chatterjee, Statistical origins of fractional derivatives in viscoelasticity, Journal of Sound and Vibration 284(5) (2005), 1239-1245.

[28] A.H. Jimenez, J.H. Santiago, A.M. Garcia and J.S. Gonzalez, Relaxation modulus in PMMA and PTFE fitting by fractional Maxwell model, Polymer Testing 21 (2002), 325-331.

[29] E. Goldfain, Fractional dynamics and standard model for particle physics, Communications in Nonlinear Science and Numerical Simulation 13 (2008), 1397-1404.

[30] O.P. Agrawal, Analytical solution for stochastic response of a fractionally damped beam, Journal of Vibration and Acoustics 126 (2004), 561-566.

[31] S.S. Ray, B.P. Poddar and R.K. Bera, Analytical solution of a dynamic system containing fractional derivative of order one-half by Adomian decomposition method, Journal of Applied Mechanics 72 (2005), 290-295.

[32] Z. Liang and X. Tang, Analytical solution of fractionally damped beam by Adomian decomposition method, Applied Mathematics and Mechanics 28(2) (2007), 219-228.

[33] I. Podlubny, Fractional Differential Equations. Academic Press, 1999.

[34] S. Adhikari, Qualitative dynamic characteristics of a non-viscously damped oscillator, Proceedings of the Royal Society of London, Series A 461(2059) (2005), 2269-2288.

[35] M.I. Friswell, S. Adhikari and Y. Lei, Non-local Finite Element Analysis of damped beams, International Journal of Solids and Structures 44(22-23) (2007), 7564-7576.

[36] S.S. Rao, Mechanical Vibrations, Addison Wesley, 1995.

[37] Y. Luke, The Special Functions and Their Approximations, Volumes 1 and 2. Academic Press, 1969.

[38] R.L. Bagley and P.J. Torvik, On the appearance of the fractional in the behavior of real material, Journal of Applied Mechanics 51 (1984), 294-298.

[39] R.C. Hibbeler, Mechanics of Materials, Printics Hall, 2003. 

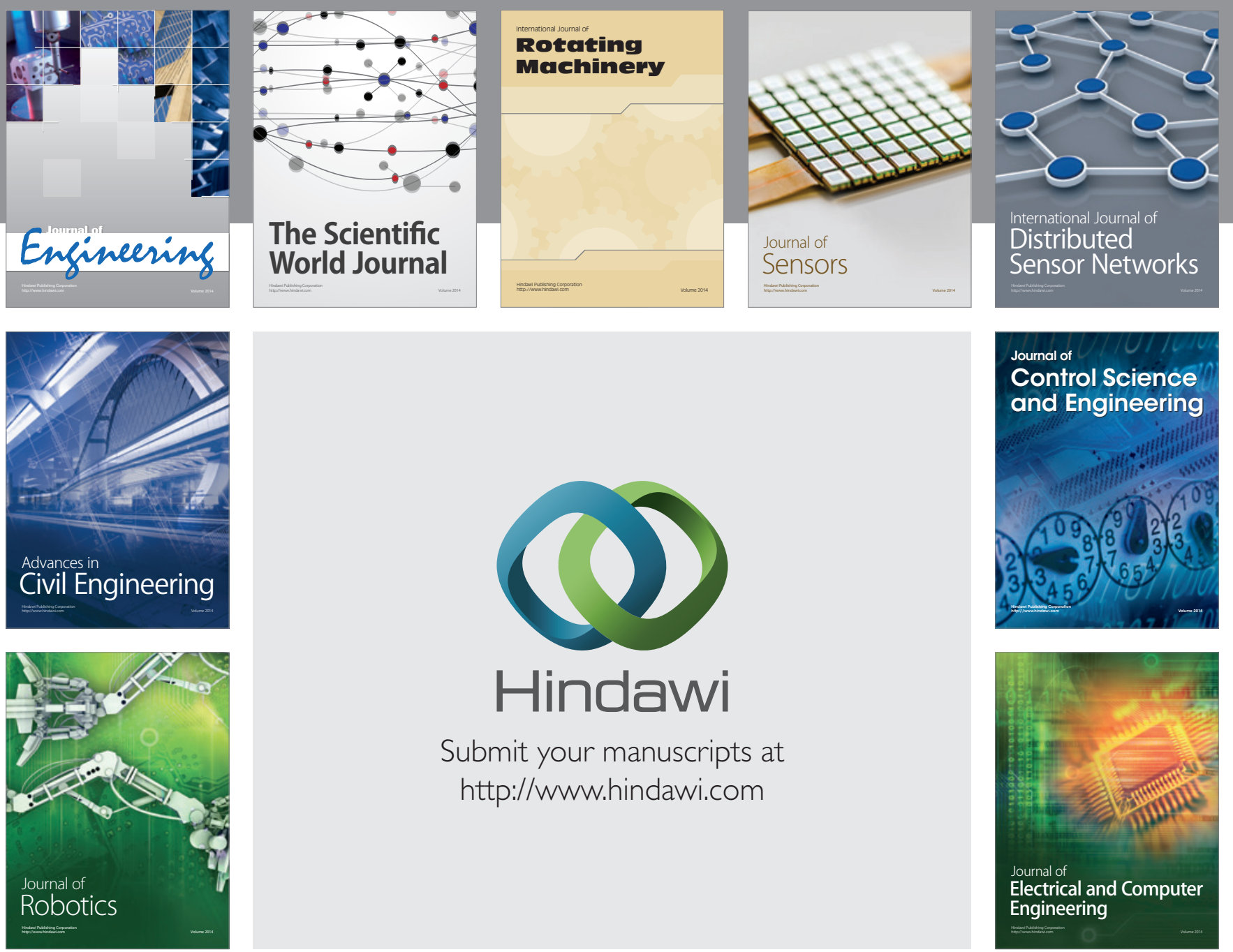

Submit your manuscripts at

http://www.hindawi.com
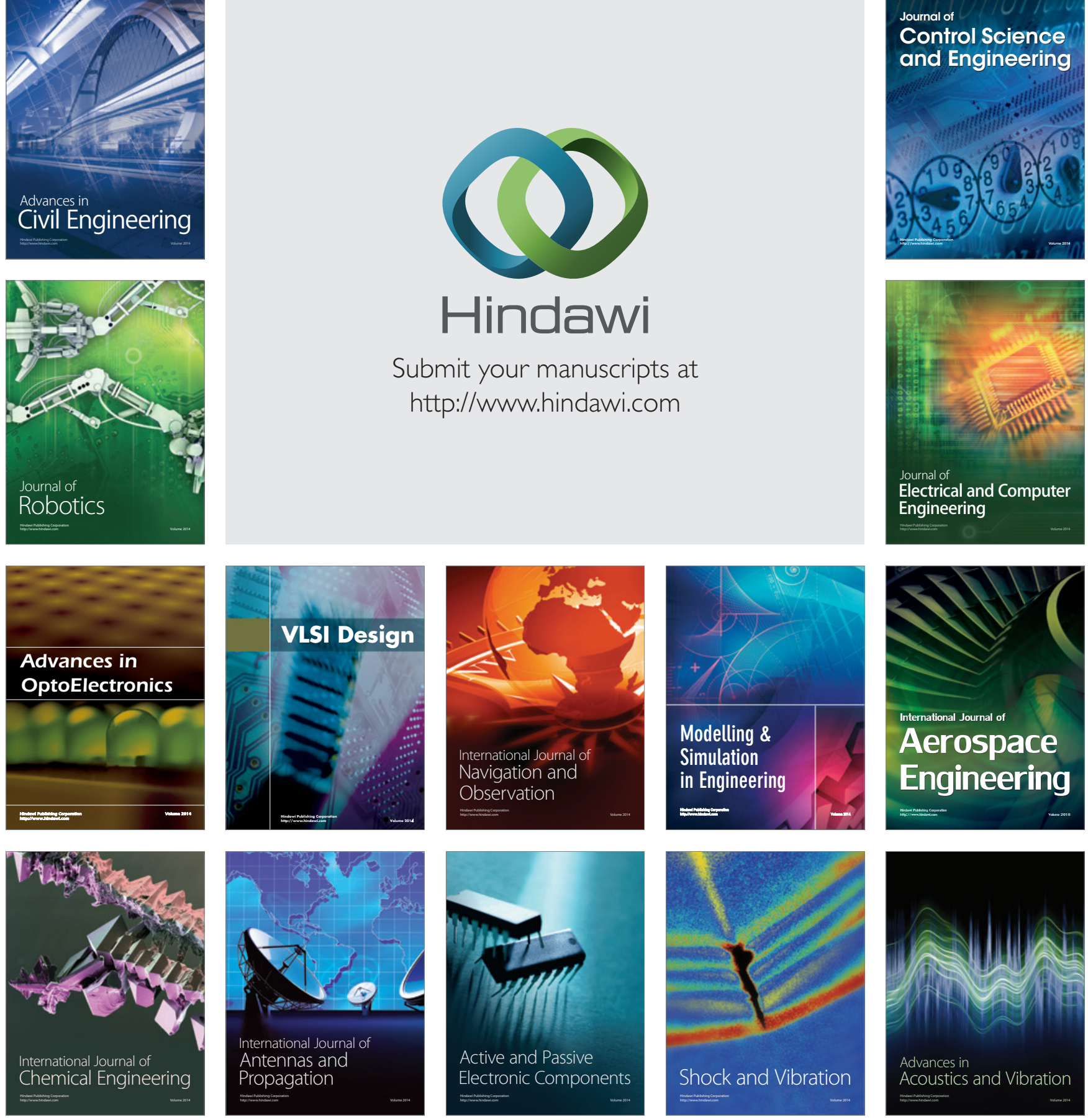